\title{
ELECTROMAGNETIC PROPERTIES OF MESOSCOPIC CYLINDER
}

\author{
E. Zipper, M. Stebelski aNd M. Lisowski \\ Instytut Fizyki, Uniwersytet Śląski \\ Uniwersytecka 4, 40-007 Katowice, Poland
}

\begin{abstract}
The electromagnetic response of a mesoscopic cylinder made of a normal metal or a semiconductor is studied. The relation between the induced current $J(q, w)$ and the electric field $E(q, w)$ is derived. It is shown that the kernel $K(\boldsymbol{g}, w)$ which determines the properties of the system has a finite limit which implies infinite conductivity. The mesoscopic cylinder by virtue of its topology and small dimensions can support a persistent current. If the coherence of currents from different channels is strong enough a novel effect - the self-sustaining current can be obtained. We show that a mesoscopic multichannel system exhibits some features which bear resemblance to the superconductor.
\end{abstract}

PACS numbers: 71.30.+h, 72.10.-d

We discuss the properties of a mesoscopic cylinder of small thickness $d$ made of a normal metal or semiconductor. The circumference and height of the cylinder are denoted by $L_{x}$ and $L_{y}$ respectively $\left(d \ll L_{x}, L_{y}\right)$.

The reaction of a $1 \mathrm{D}$ mesoscopic ring of $N$ electrons to a static and time varying magnetic field and coupled to a thermal bath has been investigated in [1].

In this paper we want to discuss the electromagnetic response of a multichannel system of cylindrical geometry with $M$ channels in the length of the cylinder using the model developed in [1].

The relation between the induced current $J(q, w)$ and the electric field $E(q, w)$ is generally written as

$$
J(\boldsymbol{q}, w)=\frac{\mathrm{i} K(\boldsymbol{q}, w)}{w} E(\boldsymbol{q}, w) .
$$

It is the form of the kernel $K(q, w)$ that determines the properties of the system both static and for finite frequencies. The following limits imply:

$$
\begin{aligned}
& \lim _{w \rightarrow 0} \lim _{\boldsymbol{q} \rightarrow 0} K(\boldsymbol{q}, w) \neq 0-\text { infinite conductivity, } \\
& \lim _{\boldsymbol{q} \rightarrow 0} \lim _{w \rightarrow 0} K(\boldsymbol{q}, w) \neq 0-\text { Meissner effect. }
\end{aligned}
$$

For normal electrons in macroscopic metallic or semiconducting samples both limits are zero. 
In this paper we calculate the conductivity $\sigma(w)$ and we study the first limit only as we are dealing with a narrow cylinder.

We show that (2) is fulfilled in the nonsuperconducting mesoscopic cylinder and its magnitude depends on the shape of the Fermi surface (FS).

The Hamiltonian in the tight-binding approximation takes a form

$$
\begin{aligned}
\mathcal{H}= & \sum_{n m}\left[\left(2 t+V_{n m}\right) c_{n m}^{+} c_{n m}-t \mathrm{e}^{\mathrm{i} \Theta_{n n+1}} c_{n+1 m}^{+} c_{n m}\right. \\
& \left.-t \mathrm{e}^{-\mathrm{i} \Theta_{n n+1}} c_{n m}^{+} c_{n+1 m}-t c_{n m+1}^{+} c_{n m}-t c_{n m}^{+} c_{n m+1}\right],
\end{aligned}
$$

where the phase of a hopping amplitude in $x$ direction comes from the magnetic flux $\phi$ of the magnetic field parallel to the cylinder axis

$$
\Theta_{n n+1}=\frac{e \phi}{\hbar c N}
$$

$\phi$ is the sum of the external flux $\phi_{\mathrm{e}}$ and flux coming from the currents

$$
\phi=\phi_{\mathrm{e}}+\mathcal{L} I(\phi),
$$

where $\mathcal{L}$ is the self-inductance coefficient, $I$ is the current. We introduce a small time-varying flux parallel to the cylinder axis $\phi \rightarrow \phi+\delta \phi(t)$

$$
\begin{aligned}
& \mathcal{H}_{1}(t)=-\frac{1}{c} I_{\mathrm{p}} \delta \phi(t) \\
& I_{\mathrm{p}}=\mathrm{i} \frac{e \hbar}{2 m a L_{x}} \sum_{n m}\left(\mathrm{e}^{\mathrm{i} \Theta_{n n+1}} c_{n+1 m}^{+} c_{n m}+\text { h.c. }\right) .
\end{aligned}
$$

$I_{\mathrm{p}}$ is the paramagnetic current running along the ring circumference. Calculating the induced current $I_{\text {ind }}$ we can find the conductivity

$$
J(w)=\frac{L_{x}}{V} I_{\text {ind }}(w)=\sigma(w) E(w),
$$

where

$$
E(w)=\frac{\mathrm{i} w}{c} \frac{\delta \phi}{L_{x}}
$$

(details are presented in [2]). Our calculations show that

$$
\lim _{w \rightarrow 0} w \operatorname{Im} \sigma(w)=-\frac{c}{V} \frac{\partial I}{\partial \phi}
$$

where

$$
I \equiv \operatorname{Tr} \rho_{0} I_{\mathrm{p}}=-c \sum_{\alpha} f_{\alpha} \frac{\partial \mathcal{E}_{\alpha}}{\partial \phi}
$$

is the equilibrium current, $\alpha$ denotes numbers of the eigenstates of $\mathcal{H}$, where $f_{\alpha}=1 /\left(\mathrm{e}^{\beta\left(\mathcal{E}_{\alpha}-\mu_{0}\right)}+1\right)$ is the Fermi-Dirac distribution function.

It follows from the basic theory of conductivity [3] that coherent electrons i.e. those which run without scattering obey the following relation:

$$
\lim _{w \rightarrow 0} w \operatorname{Im} \sigma(w)=\frac{n_{c} e^{2}}{m}
$$


where $n_{c}$ is the density of coherent electrons. Thus coherent electrons, absent in macroscopic samples, exist in mesoscopic cylinders and they run persistent currents. Comparing Eqs. (11) with (2) we see that

$$
\lim _{w \rightarrow 0} \lim _{q \rightarrow 0} K(q, w)=-\frac{c}{V} \frac{\partial I}{\partial \phi},
$$

is equivalent to a free acceleration of electrons in the mesoscopic system.

Let us discuss now the equilibrium current (12). The amplitude of this current which runs in the absence of an electric field depends on the size of the sample and on the FS geometry. The magnitude of the current depends on the strength of the phase correlations between currents of different channels.

For $M$ channel system we can express the current as a Fourier series [4]

$$
\begin{aligned}
I(\phi) & =\exp \left(-\frac{L_{x}}{2 \lambda}\right) \sum_{m=1}^{M} \sum_{l=1}^{\infty} \frac{4 T}{\pi T^{*}} \frac{2 e t}{N \hbar} \frac{\exp \left(-l T / T^{*}\right)}{1-\exp \left(-2 l T / T^{*}\right)} \\
& \times \sin \left[k_{F_{x}}(m) a\right] \cos \left[l N k_{F_{x}}(m) a\right] \sin \left(\frac{2 \pi l \phi}{\phi_{0}}\right),
\end{aligned}
$$

where $k_{F_{x}}$ is calculated from the equation for the FS, $\lambda$ is the mean free path, $T^{*}$ is a characteristic temperature defined by the energy gap, $\phi_{0}=h c / e$.

For certain values of parameters the current is paramagnetic, for others it is diamagnetic. In the following we discuss the diamagnetic solutions (for paramagnetic solutions see [5]).

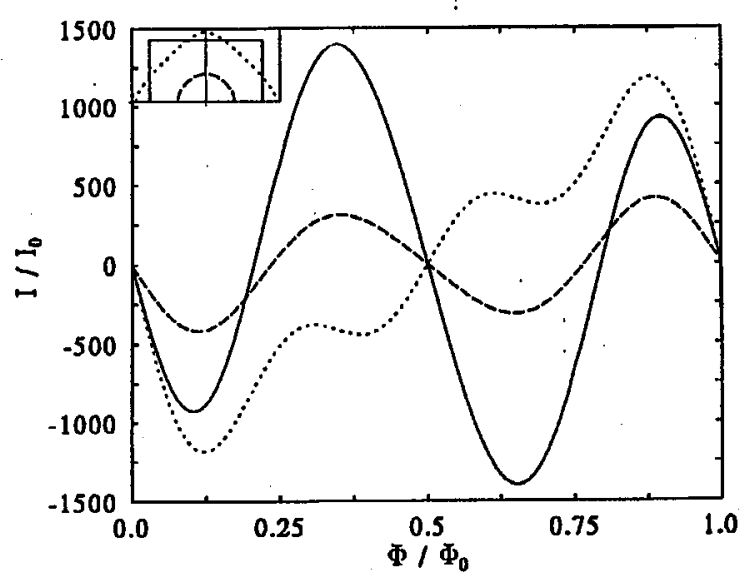

Fig. 1. Persistent currents vs. flux for different shapes of the Fermi surfaces: rectangular (solid line), triangular with rounded corners (dotted line) and circular (dashed line). In the inserted figure the shapes of the Fermi surfaces are shown.

Figure 1 shows a persistent current for three different shapes of the FS (inserted figure) for $T=1 \mathrm{~K}$. We see that the amplitude of the persistent current increases with increasing departure of the FS from circular shape because the phase correlation of the channel currents increases. 

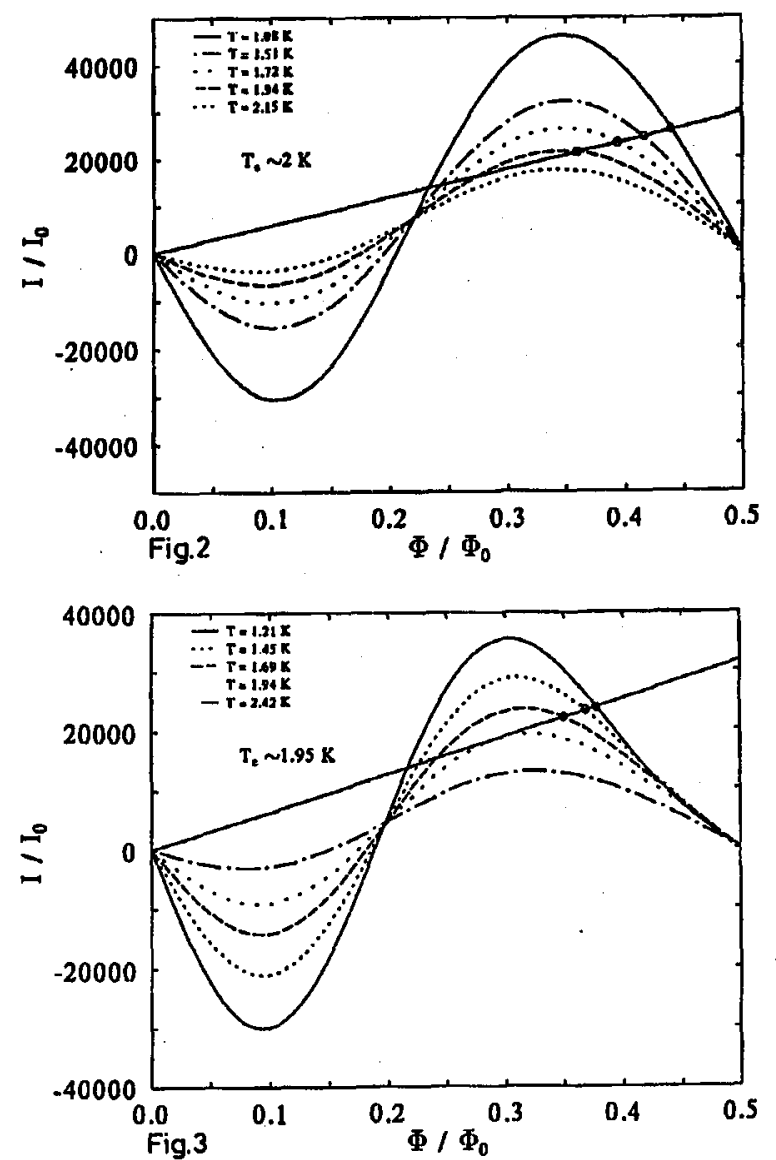

Fig. 2. The graphical solution of a set of self-consistent Eqs. (15) and (6) for different temperatures and for the rectangular Fermi surface. The nonzero crossings of the straight line (6) with the current-flux characteristic (15) denoted by circles correspond to the flux trapped in the mesoscopic cylinder.

Fig. 3. The graphical solution of a set of self-consistent Eqs. (15) and (6) for different temperatures and for the triangular with rounded corners Fermi surface. The nonzero crossings of the straight line (6) with the current-flux characteristic (15) denoted by circles correspond to flux trapped in the mesoscopic cylinder.

Let us consider a cylinder which consists of a set of concentric sheets. The magnetic flux $\phi$ which drives the current (15) is given by (6). The graphical solutions of the self-consistent Eqs. (15) and (6) for the zero external field $\left(\phi_{\mathrm{e}}=0\right)$ and Fermi surfaces being rectangular and triangular with rounded corners are presented in Fig. 2 and Fig. 3, respectively. The intersections of the two curves, marked by circles give the values of self-sustaining currents. They correspond to flux trapping in the cylinder. The temperature at which the self-consistent solution 
appears is denoted by $T_{\mathrm{c}}$. For circular FS we do not observe flux trapped in the system because the current is too small.

In conclusion, we have shown that a mesoscopic cylinder made of a metal or semiconductor exhibits some features being a manifestation of the quantum coherence.

The electromagnetic kernel in the long wave length limit and the low frequency limit is finite which is equivalent to the infinite conductivity. Thus, although the dissipation was taken into account, a part of the electrons is in a coherent state.

The mesoscopic cylinder runs the persistent current which, for the FS sufficiently flat, can become self-sustaining which is equivalent to the flux trapping. All these features bear some resemblance to the characteristics of superconductors.

The work was supported by grant of the Committee for Scientific Research No. PB 1108/P03/95/08.

\section{References}

[1] N. Trivedi, D.A. Browne, Phys. Rev. B 38, 9581 (1988).

[2] M. Stebelski, M. Lisowski, E. Zipper, to be published.

[3] N.W. Ashcroft, N.D. Mermin, Solid State Physics, PWN, Warszawa 1986.

[4] H.F. Cheung, Y. Gefen, E.K. Riedel, IBM J. Res. Develop. 32, 359 (1988).

[5] M. Stebelski, M. Szopa, E. Zipper, Z. Phys. B 103, 79 (1997). 